Supporting Information for:

\title{
Starting from a Fixed Geometry: Real-Time XPS Investigation of a Surface Reaction with Controlled Molecular Configurations
}

Timo Glaser ${ }^{1}$, Christian Länger ${ }^{1}$, Julian Heep ${ }^{1}$, Jannick Meinecke ${ }^{2}$, Mathieu G. Silly ${ }^{3}$, Ulrich Koert ${ }^{2}$, and Michael Dürr ${ }^{1, *}$

${ }^{1}$ Institut für Angewandte Physik and Zentrum für Materialforschung, Justus-Liebig-Universität Giessen, Heinrich-Buff-Ring 16, 35392 Giessen, Germany

${ }^{2}$ Fachbereich Chemie, Philipps-Universität Marburg, Hans-Meerwein-Straße 4, 35032 Marburg, Germany

${ }^{3}$ Synchrotron SOLEIL, L’Orme des Merisiers, Saint Aubin, Gif sur Yvette 91192, France

*Corresponding author: michael.duerr@ap.physik.uni-giessen.de (M.D.) 
This Supporting Information includes

(I) Direct current heating ramp and heating rate (Figs. S1(a) and (b))

(II) C 1s spectra of cyclooctyne ether on $\mathrm{Si}(001)$ (Fig. S2)

(III) Applicability of Arrhenius law for the reaction rate $R$

1. Potential energy curve and schematics for surface reaction of covalently attached cyclooctyne ether on $\mathrm{Si}(001)$ (Fig. S3)

2. Rate equations - temperature dependence of reaction rate $R$ (Eqs. 1-8) 
I Direct current heating ramp and heating rate

(a)

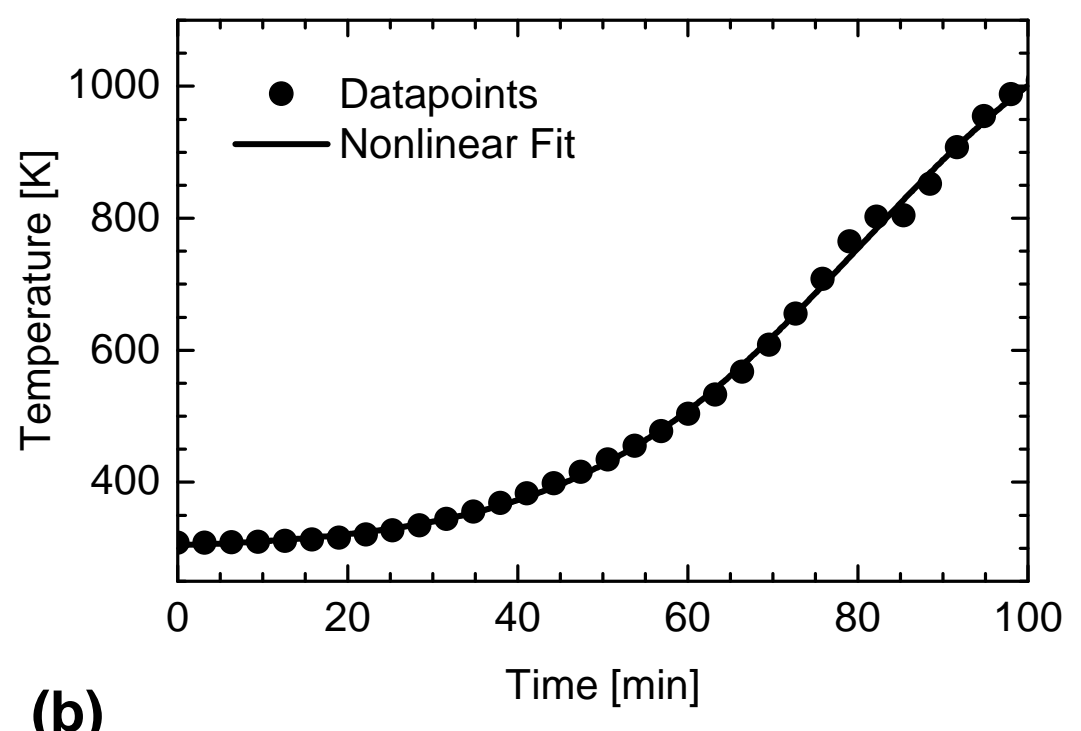

(b)

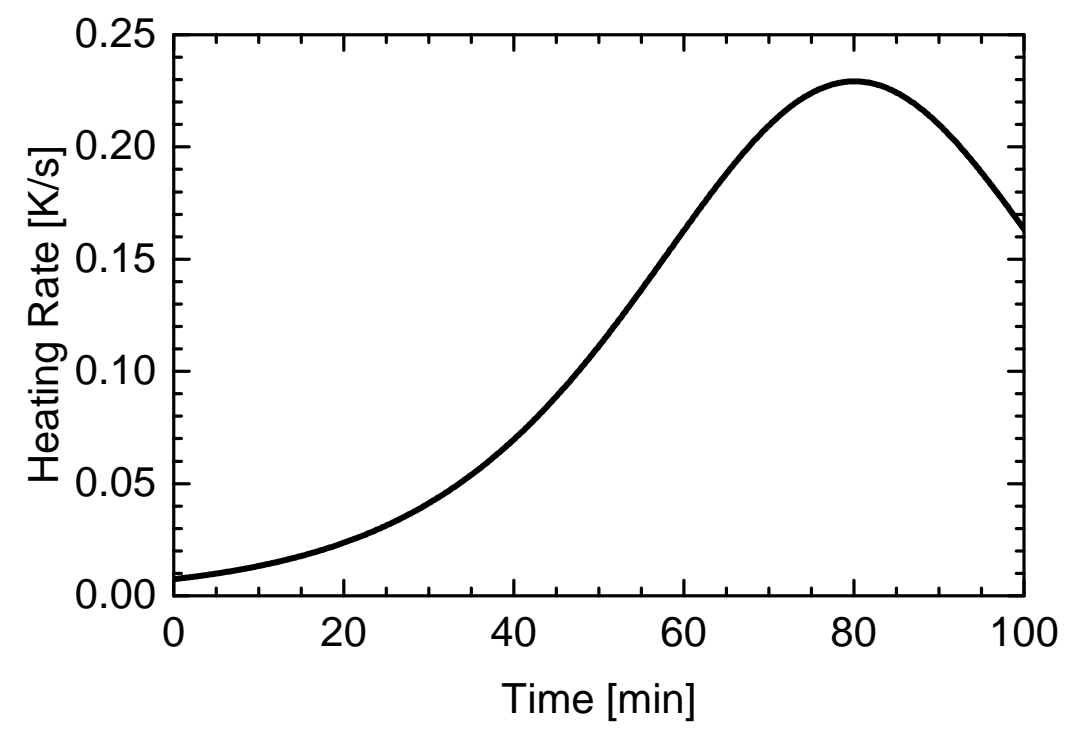

Figure S1: (a) Sample temperature as a function of time during a direct current heating ramp from $I=0 \mathrm{~A}$ to $1.2 \mathrm{~A}$ with an increase of $0.2 \mathrm{~mA} / \mathrm{s}$ measured with a thermocouple attached to a reference sample. The data points were fitted with a tanh-function in order to calculate the heating rate. (b) Calculated heating rate as a function of time. The maximum heating rate was $0.23 \mathrm{~K} / \mathrm{s}$ at about $800 \mathrm{~K}$. 


\section{1s spectra of cyclooctyne ether on $\mathrm{Si}(001)$}

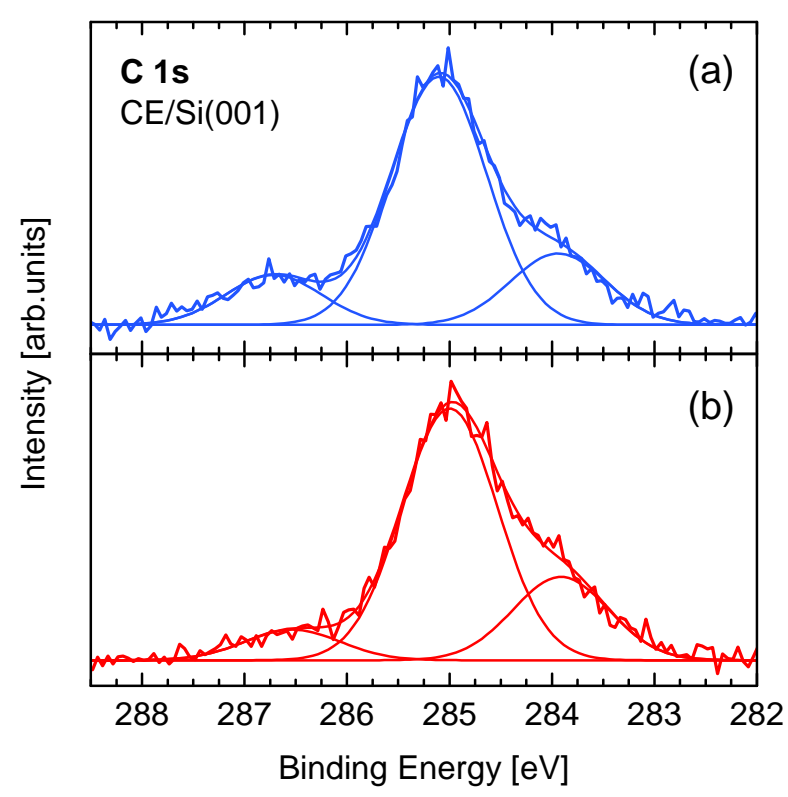

Figure S2: C 1s spectra of cyclooctyne ether on $\operatorname{Si}(001)$. In (a), adsorption and measurement were performed close to room temperature $\left(T_{\mathrm{S}}=310 \mathrm{~K}\right)$. Three peaks are identified which are assigned to (i) carbon atoms bound to an oxygen atom $(286.7 \mathrm{eV})$, (ii) carbon atoms with carbon and hydrogen as binding partners $(285.1 \mathrm{eV})$, and (iii) carbon atoms with one covalent bond to an Si atom $(283.9 \mathrm{eV})$. The ratio of the integrated intensity of the three peaks is 2:8:2 in perfect agreement with the cyclooctyne ether molecule being covalently bound to the silicon surface with two carbon atoms, thus comprising an intact ether group with two carbon atoms in direct neighborhood of the oxygen atom and further eight remaining carbon atoms with carbon-carbon and carbon-hydrogen bonds only. The somewhat larger width of the peak associated with the C-C-C peak is interpreted in terms of the different next-next neighbors of the respective $\mathrm{C}$ atoms. The spectrum in (b) was taken during a heating ramp at $T_{\mathrm{S}}=505 \mathrm{~K}$. The total intensity is the same as for the spectrum in (a), however, the intensity of the peak at $286.7 \mathrm{eV}$ (carbon atoms bound to an oxygen atom) is reduced by the same amount as the intensity of the peak at $283.9 \mathrm{eV}$ (carbon atoms with one covalent bond to an Si atom) is increased. This change in intensity is expected for ether cleavage of $\mathrm{CE}$ on $\mathrm{Si}(001)$ : during ether cleavage, one carbon bound to oxygen is converted into one carbon bound to silicon. No further reactions of CE can be deduced from this change of the spectra. 


\section{Applicability of Arrhenius law for the reaction rate $R$}

III.1 Potential energy curve and schematics for surface reaction of covalently attached cyclooctyne
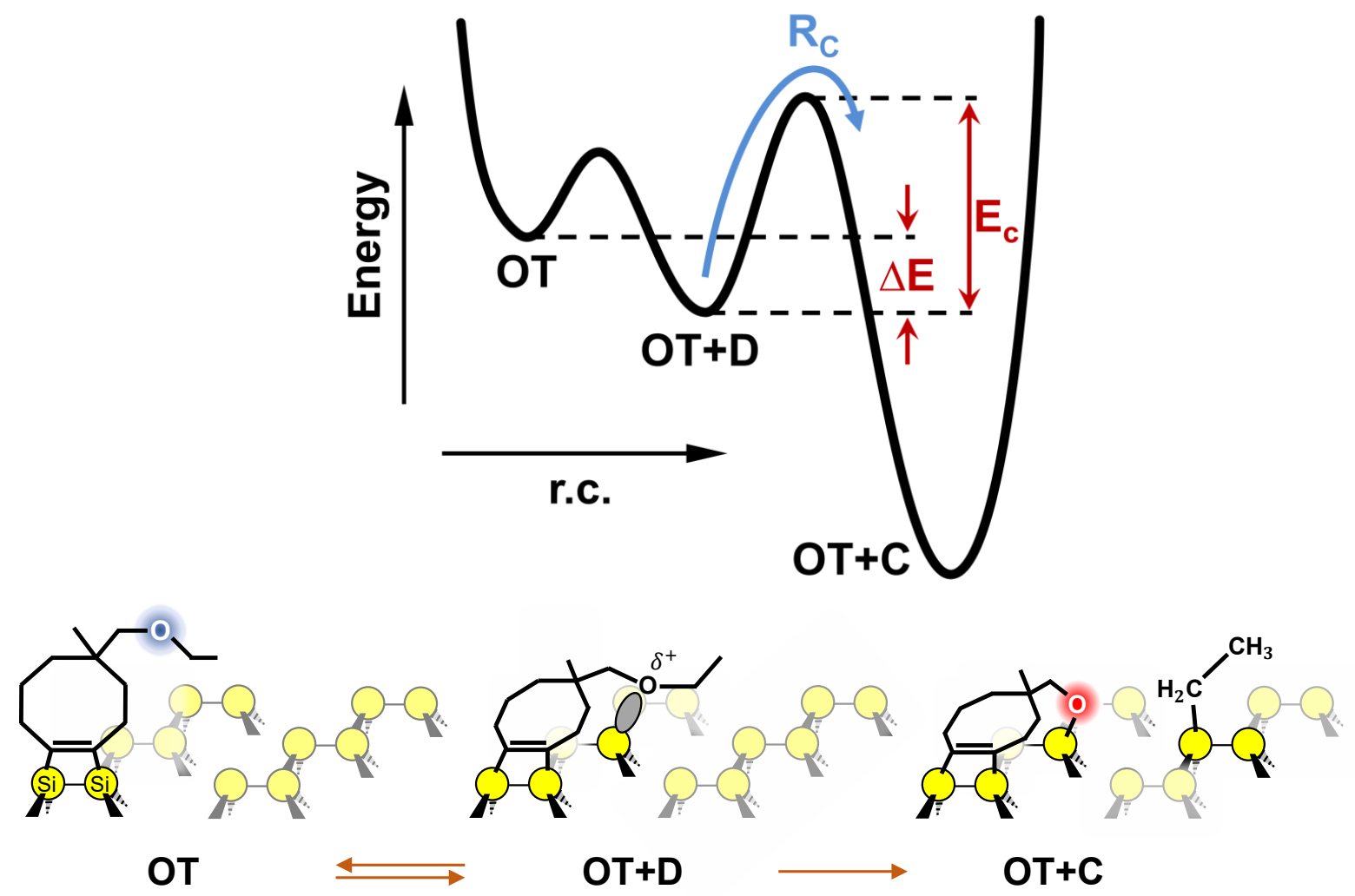

Figure S3: Top: Schematic potential energy curve for the activated reaction of CE on $\mathrm{Si}(001)$.

Reaction rate $R_{\mathrm{C}}$ from $\mathrm{OT}+\mathrm{D}$ to $\mathrm{OT}+\mathrm{C}$ is depicted by the blue arrow. Energies are highlighted in red. Bottom left: Intact cyclooctyne ether adsorbed via the former strained triple bond on top of one dimer (OT), the ether group is intact (blue oxygen atom). Center: The oxygen of the ether group forms a dative bond (grey) with a neighbored Si dimer $(\mathrm{OT}+\mathrm{D})$. Right: The ether group has reacted with two $\mathrm{Si}$ dimers via ether cleavage $(\mathrm{OT}+\mathrm{C}$, red oxygen atom). 
III.2 Rate equations - temperature dependency of reaction rate $R$

Reaction rate $R_{\mathrm{C}}$ from intermediate $(\mathrm{OT}+\mathrm{D})$ into the final state $(\mathrm{OT}+\mathrm{C})$ :

$$
R_{\mathrm{C}}=\theta_{\mathrm{OT}+\mathrm{D}} \cdot k_{\mathrm{C}}=\theta_{\mathrm{OT}+\mathrm{D}} \cdot A_{\mathrm{C}} \cdot \exp \left(-\frac{E_{\mathrm{C}}}{k_{\mathrm{B}} T}\right)
$$

- $R_{\mathrm{C}}=$ Reaction rate for the reaction from $\mathrm{OT}+\mathrm{D}$ to $\mathrm{OT}+\mathrm{C}$

- $\theta_{\mathrm{OT}+\mathrm{D}}=$ Rel. number (coverage) of intermediate states $\mathrm{OT}+\mathrm{D}$

- $k_{\mathrm{C}}=$ Reaction rate constant for the reaction from OT+D to OT+C

- $A_{\mathrm{C}}=$ Pre-exponential factor for the reaction from $\mathrm{OT}+\mathrm{D}$ to $\mathrm{OT}+\mathrm{C}$

- $E_{\mathrm{C}}=$ Energy barrier for the reaction from $\mathrm{OT}+\mathrm{D}$ to $\mathrm{OT}+\mathrm{C}$

- $k_{\mathrm{B}}=$ Boltzmann constant

- $T=$ Temperature

In order to find an expression for $\theta_{\mathrm{OT}+\mathrm{D}}$, we take a look at the forward and reverse reaction of initial $(\mathrm{OT})$ and intermediate state $(\mathrm{OT}+\mathrm{D})$ :

$$
\mathrm{OT} \underset{k_{\mathrm{r}}}{\stackrel{k_{\mathrm{f}}}{\rightleftharpoons}} \mathrm{OT}+\mathrm{D}
$$

- $k_{\mathrm{f}}=$ reaction rate constant for the forward reaction from OT to OT $+\mathrm{D}$

- $k_{\mathrm{r}}=$ reaction rate constant for the reverse reaction from $\mathrm{OT}+\mathrm{D}$ to $\mathrm{OT}$

With a low barrier between $\mathrm{OT}$ and $\mathrm{OT}+\mathrm{D}$, we assume an equilibrium between the two states and thus:

$$
\frac{\theta_{\mathrm{OT}+\mathrm{D}}}{\theta_{\mathrm{OT}}}=\frac{k_{\mathrm{f}}}{k_{\mathrm{r}}}=\frac{A_{\mathrm{f}}}{A_{\mathrm{r}}} \cdot \exp \left(\frac{\Delta E}{k_{\mathrm{B}} T}\right)
$$




$$
\Rightarrow \quad \theta_{\mathrm{OT}+\mathrm{D}}=\theta_{\mathrm{OT}} \cdot \frac{A_{\mathrm{f}}}{A_{\mathrm{r}}} \cdot \exp \left(\frac{\Delta E}{k_{\mathrm{B}} T}\right)
$$

- $\theta_{\mathrm{OT}}=$ Rel. number (coverage) of initial states OT

- $A_{\mathrm{f}}=$ Pre-exponential factor for the forward reaction from OT to OT $+\mathrm{D}$

- $A_{\mathrm{r}}=$ Pre-exponential factor for the reverse reaction from $\mathrm{OT}+\mathrm{D}$ to $\mathrm{OT}$

- $\Delta E=$ Energy difference between initial $(\mathrm{OT})$ and intermediate state $(\mathrm{OT}+\mathrm{D})$

Combination of Eq. 4 with Eq.1 leads to an expression similar to the Arrhenius equation:

$$
\begin{gathered}
R_{\mathrm{C}}=\theta_{\mathrm{OT}} \cdot \frac{A_{\mathrm{f}}}{A_{\mathrm{r}}} \cdot A_{\mathrm{C}} \cdot \exp \left(-\frac{E_{\mathrm{C}}}{k_{\mathrm{B}} T}\right) \cdot \exp \left(\frac{\Delta E}{k_{\mathrm{B}} T}\right) \\
\Rightarrow \quad R_{\mathrm{C}}=\theta_{\mathrm{OT}} \cdot \frac{A_{\mathrm{f}}}{A_{\mathrm{r}}} \cdot A_{\mathrm{C}} \cdot \exp \left(-\frac{E_{\mathrm{C}}-\Delta E}{k_{\mathrm{B}} T}\right)
\end{gathered}
$$

Assuming $R_{\mathrm{C}}$ to be the rate limiting step for the total reaction rate $R_{\text {total }}$ from OT to OT $+\mathrm{C}$, we can write

$$
\begin{gathered}
R_{\text {total }}=\theta_{\mathrm{OT}} \cdot k_{\text {total }} \approx R_{\mathrm{C}}=\theta_{\mathrm{OT}} \cdot \frac{A_{\mathrm{f}}}{A_{\mathrm{r}}} \cdot A_{\mathrm{C}} \cdot \exp \left(-\frac{E_{\mathrm{C}}-\Delta E}{k_{\mathrm{B}} T}\right) \\
\Rightarrow \quad k_{\text {total }}=\frac{A_{\mathrm{f}}}{A_{\mathrm{r}}} \cdot A_{\mathrm{C}} \cdot \exp \left(-\frac{E_{\mathrm{A}}}{k_{\mathrm{B}} T}\right)
\end{gathered}
$$

- $R_{\text {total }}=$ Reaction rate for the reaction from $\mathrm{OT}$ to $\mathrm{OT}+\mathrm{C}$

- $k_{\text {total }}=$ Reaction rate constant for the reaction from OT to OT $+\mathrm{C}$

As a result, $k_{\text {total }}$ depends only on the overall energy barrier $E_{\mathrm{A}}=E_{\mathrm{C}}-\Delta E$. 